\title{
Cambios hormonales asociados al embarazo. Afectación gingivo-periodontal
}

\author{
FIGUERO-RUIZ E* \\ PRIETO PRIETO I** \\ BASCONES-MARTÍNEZ A***
}

\begin{abstract}
Figuero-Ruiz E, Prieto Prieto I, Bascones-Martínez A. Cambios hormonales asociados al embarazo. Afectación gingivo-periodontal. Av Periodon Implantol. 2006; 18, 2: 101-113.
\end{abstract}

\begin{abstract}
RESUMEN
La gingivitis gravídica es una inflamación proliferativa, vascular e inespecífica con un amplio infiltrado inflamatorio celular. Clínicamente se caracteriza por una encía intensamente enrojecida que sangra fácilmente, por un engrosamiento del margen gingival y por hiperplasia de las papilas interdentales que pueden dar lugar a la aparición de pseudobolsas. La prevalencia varía entre el 35 y el $100 \%$ de las embarazadas con gingivitis previa.
\end{abstract}

Aunque parece que durante el embarazo la susceptibilidad de los tejidos gingivales a la inflamación está relacionada con los cambios hormonales propios del mismo, el mecanismo exacto por el cual estas hormonas incrementan la inflamación gingival es desconocido. El cambio hormonal más significativo es el incremento en la producción de estrógeno y progesterona.

La respuesta de los tejidos a las hormonas sexuales femeninas va a depender del tipo de hormona circulante, de la proporción de estrógeno/progesterona, así como de la concentración tisular de la hormona. Sus efectos a nivel del tejido gingival son variados y tienden a ser clasificados en cuatro grandes grupos: cambios vasculares, cambios celulares, cambios microbiológicos y cambios inmunológicos.

\section{PALABRAS CLAVE}

Gingivitis. Embarazo. Granuloma gravídico. Estrógenos. Progesterona. Patogénesis. Diagnóstico.

Aceptado para publicación: Noviembre 2005.

\section{INTRODUCCIÓN}

El embarazo es una condición en la que se encuentra la mujer durante un periodo de unos nueve meses, desde la fecundación del cigoto hasta el parto. Se caracteriza por una serie de cambios sistémicos en diferentes niveles del organismo tales como alteraciones endocrinas, con un incremento en la producción de

* Licenciada en Odontología (UCM). Becaria de investigación del Ministerio de Educación. Postgrado de Periodoncia (UCM). Departamento de Medicina y Cirugía Bucofacial (Estomatología III). Facultad de Odontología. Universidad Complutense de Madrid. España.

** Doctor en Odontología (UCM). Postgrado de Periodoncia (UCM). Departamento de Medicina y Cirugía Bucofacial (Estomatología III). Facultad de Odontología. Universidad Complutense de Madrid. España.

*** Catedrático de Medicina Bucal y Periodoncia. Departamento de Medicina y Cirugía Bucofacial (Estomatología III). Facultad de Odontología. Universidad Complutense de Madrid. España. 
estrógenos y progesterona; cambios a nivel cardiovascular con un aumento en el volumen plasmático y en el gasto cardíaco; cambios pulmonares, caracterizados por un aumento en el volumen de aire circulante e incluso la aparición de disnea; cambios a nivel gastrointestinal, con una alteración de los hábitos alimentarios (incremento en el consumo de azúcares) y aparición de náuseas y vómitos; y cambios hematológicos, con una disminución del hematocrito, de la tasa de hemoglobina y del número de eritrocitos (1).

A nivel bucal se ha descrito la aparición de ciertas alteraciones gíngivo-periodontales, existiendo determinadas patologías propias, tales como la gingivitis del embarazo o el granuloma gravídico (2-4). La gingivitis del embarazo o gingivitis gravídica, es una inflamación proliferativa, vascular e inespecífica con un amplio infiltrado inflamatorio celular. Clínicamente se caracteriza por una encía intensamente enrojecida que sangra fácilmente por un engrosamiento del margen gingival y por hiperplasia de las papilas interdentales que pueden dar lugar a la aparición de pseudobolsas (5-8). Löe y Silness (9) en 1963 describen que los primeros síntomas aparecen en el segundo mes de embarazo y continúan hasta el octavo mes, momento a partir del cual se observa cierta mejoría para estabilizarse finalmente tras el parto. Los estudios clínicos muestran una prevalencia que varía entre el 35 y el $100 \%$ de las embarazadas con gingivitis previa. Sin embargo, su incidencia es de tan sólo 0,03\% en aquellas mujeres libres de placa al inicio del embarazo y con buenos hábitos de higiene oral durante el mismo $(9,10)$.

El granuloma gravídico, también llamado tumor del embarazo, es una reacción inflamatoria proliferativa fi- brovascular exagerada con relación a un estímulo ordinario localizada fundamentalmente en la encía (11). Se describe como una masa localizada roja o roja-amoratada, nodular o ulcerada que sangra fácilmente y que aparece frecuentemente en mujeres $(0,5-5 \%)$ en torno al segundo trimestre del embarazo y crece a lo largo del mismo alcanzando un tamaño que no suele superar los 2 $\mathrm{cm}$. Su etiología es desconocida, pero se han implicado factores traumáticos, higiénicos y hormonales. (12-14).

\section{A. CARACTERÍSTICASS GENERALES DE LASS HORMONAS SEXUALES FEMENINAS}

Se denominan hormonas sexuales a las producidas por el ovario (o el testículo) y cuyas acciones se manifiestan en el desarrollo de los caracteres sexuales secundarios y en el proceso de la reproducción (15). Pertenecen a una subclase de lípidos denominados genéricamente esteroides, que se caracterizan por tener una estructura básica común, el ciclopentanoperhidrofenantreno, que está formado por tres anillos bencénicos (A, B, C), constituidos cada uno por seis átomos de carbono y un anillo ciclopentano (D), formado por cinco átomos de carbono.

Existen tres grandes grupos de esteroides sexuales, que se diferencian entre sí por poseer cada uno un número específico de átomos de carbono:

- Con 18 átomos de carbono: el estrano, del cual derivan los estrógenos (Figura 1).

- Con 19 átomos de carbono: el androstano, del que derivan los andrógenos (Figura 2).

- Con 21 átomos de carbono: el pregnano. Derivan de él los gestágenos (Figura 3).

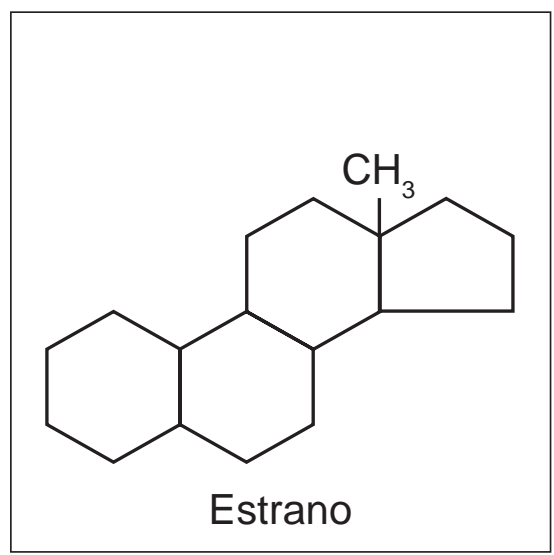

Figura 1: Estructura química del estrano. Tomada de Usandizaga (27)

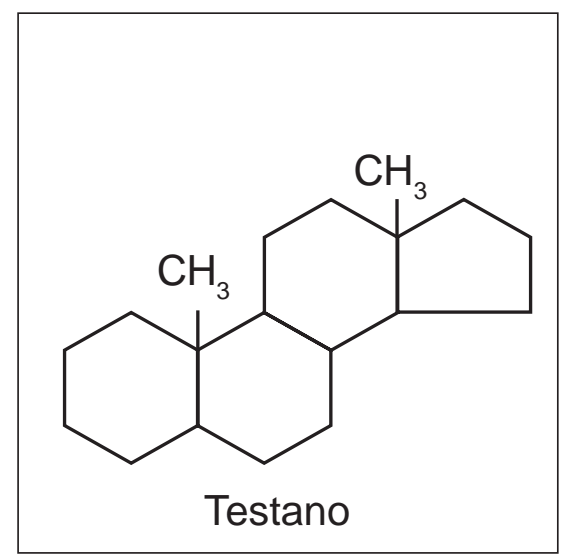

Figura 2: Estructura química del androstano. Tomada de Usandizaga (27).

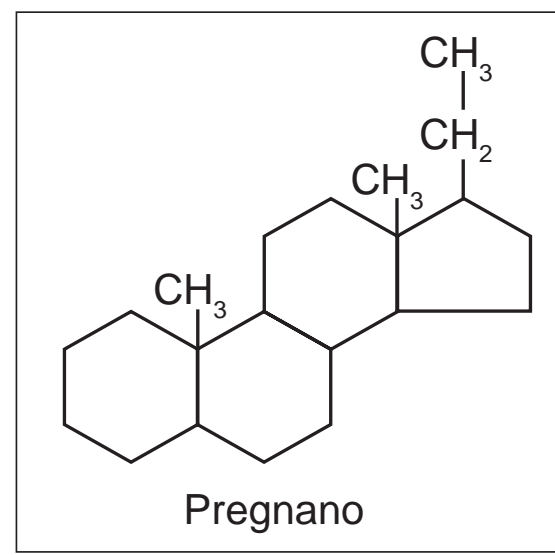

Figura 3: Estructura química del pregnano. Tomada de Usandizaga (27). 


\section{ESTRÓGENOS}

La denominación dada a estas hormonas procede de su capacidad para provocar el estro o período de celo en las hembras de los mamíferos. Además de los 18 átomos de carbono, poseen un anillo aromático (tres dobles enlaces) en el anillo A y un grupo hidróxilo en C-3. Los estrógenos más importantes fisiológicamente producidos en el ovario son el estradiol y la estrona. Existe un tercer estrógeno natural, el estriol, de muy baja potencia como estrógeno, pero de elevada producción en caso de embarazo.

El estradiol es el estrógeno que segrega el ovario en mayor cantidad y el de mayor potencia biológica (Figura 4). La producción diaria de estradiol varía según la fase del ciclo ovárico, con cifras de 30 a $50 \mathrm{mg} /$ día $(70-100 \mathrm{pg} / \mathrm{ml})$ en la fase folicular precoz, 300 a 500 $\mathrm{mg} /$ día (220-400 pg/ml) en la fase preovulatoria y 200 a $350 \mathrm{mg} /$ día (150-250 pg/ml) en la fase lútea. Tan ínfimas cantidades dan idea de su extremada potencia biológica, sobre todo al considerar que sólo una pequeña cantidad circula libre en sangre.

La estrona es el principal estrógeno posmenopáusico. Es menos potente que el estradiol y no presenta cambios cíclicos.

Los estrógenos son los responsables de la aparición y mantenimiento de los caracteres sexuales primarios durante la etapa puberal. Además, a corto plazo, son responsables directos de todas las transformaciones cíclicas que tienen lugar durante la fase folicular, en especial el grosor del endometrio y el moco cervical. También desarrollan su acción sobre el hipotálamo, la

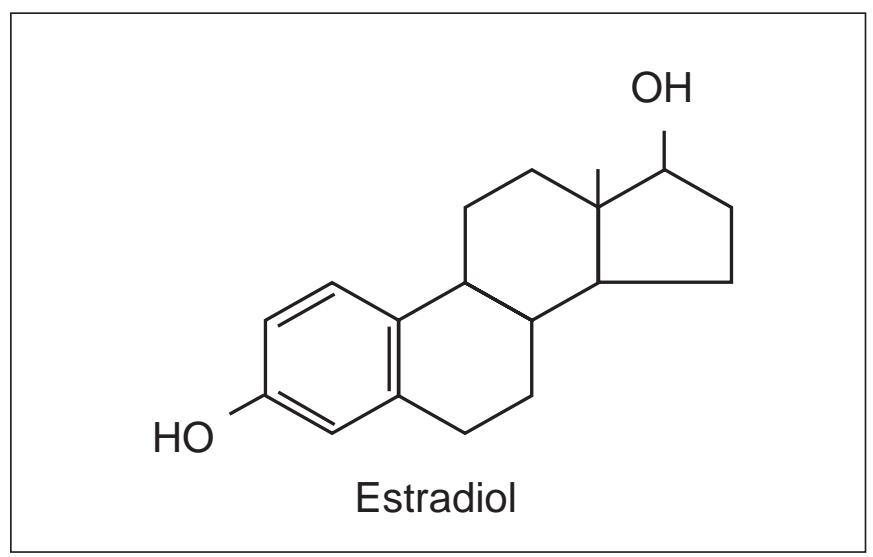

Figura 4: Estructura química del estradiol. Tomada de Usandizaga (27). hipófisis, el tiroides y la glándula suprarrenal. Son importantes en el mantenimiento del sistema simpático y parasimpático, intervienen en el equilibrio hidrosalino (retención de sodio y agua), en el metabolismo del calcio y del fósforo (limitando la reabsorción ósea), en el metabolismo hidrocarbonado y lipídico, actúan sobre la circulación y coagulación sanguínea y mantienen el trofismo de la piel femenina $(8,15,16)$.

\section{GESTÁGENOS O PROGESTÁGENOS}

Los principales gestágenos producidos en el ovario son la pregnenolona y la progesterona. La pregnenolona es un precursor de todos los esteroides sintetizados en el ovario. La progesterona es producida en grandes cantidades por el cuerpo lúteo y la placenta (25 $\mathrm{mg} /$ día; 8-30 ng/ml) (Figura 5). Posee una actividad biológica pequeña, lo que, unido a que tiene una vida media muy corta, justifica la presencia de tan grandes cantidades de esta hormona para ejercer su efecto. Su misión fundamental es la protección del embarazo en sus fases iniciales. Tiene también una acción termogénica importante, al producir una elevación de la temperatura en medio grado en la fase lútea del ciclo; una acción metabólica (retención de agua y sodio) y actúa sobre el crecimiento y el trofismo de la mama en colaboración con los estrógenos $(15,16)$.

\section{B. CICLO GENITAL FEMENINO}

Se entiende por ciclo genital femenino a la serie de fenómenos anatomofisiológicos que, desde la pubertad hasta la menopausia, se repiten periódica y regu-

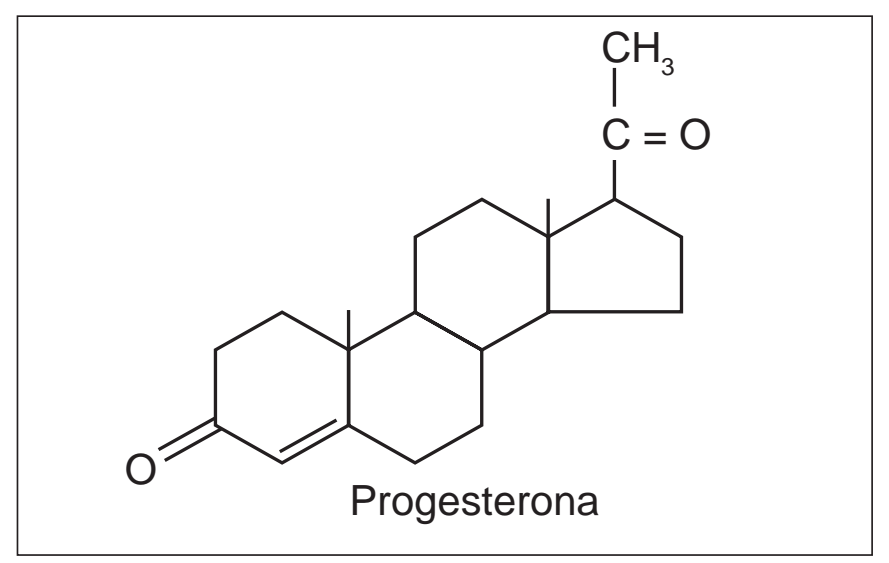

Figura 5: Fórmula química de la progesterona. Tomada de Usandizaga (27) 
larmente en el organismo de la mujer, muy especialmente en su aparato genital. Este proceso se repite de forma regular durante toda la etapa de madurez sexual, estando regido por estructuras superiores en el hipotálamo y la hipófisis.

El fenómeno fundamental del ciclo es la ovulación, que tiene lugar en el ovario. Tiene una función doble: liberar células germinales capaces de ser fecundadas y secretar hormonas que van a tener una amplia repercusión en todo el organismo.

La formación de los estrógenos en los ovarios comienza entre la octava y la décima semana de gestación del feto, y las oogonias se transforman en oocitos primarios entre las semanas diez y once. Un número finito de estas células germinales se concentra en el ovario con un máximo de siete millones de oocitos entre el quinto y el sexto mes de gestación. Posteriormente, a través del proceso de atresia, las células germinales decrecen en número hasta alcanzar el millón de folículos primordiales conteniendo un único óvulo cada uno en el momento del nacimiento. La mayoría de estos folículos no alcanzan su desarrollo, llegando a la pubertad unos 400.000 . Será en este momento cuando la maduración final de estas células comience (8).

El ciclo ovárico puede ser dividido en dos fases: maduración folicular y fase de cuerpo lúteo $(8,15-17)$ (Figura 6).

\section{FASE DE MADURACIÓN FOLICULAR}

El primer signo del inicio del desarrollo folicular es la transformación de las células planas que rodean al oocito en células cúbicas, que proliferarán para dar lugar a un estrato denominado membrana granulosa. Por fuera de esta membrana las células del estroma ovárico inician una diferenciación, aumentan su volumen y su vascularización para formar la teca interna. Esta estructura se denomina folículo primario o preantral.

Entre las células de la granulosa comienzan a aparecer pequeñas vacuolas, que, al confluir, pasan a formar una cavidad llena de líquido transparente denominada antro folicular o cavidad antral, pasando a ser folículos secundarios o antrales.

La mayoría de estos folículos van a sufrir un proceso degenerativo e involutivo (atresia), de tal manera que sólo uno de los folículos continua su crecimiento para

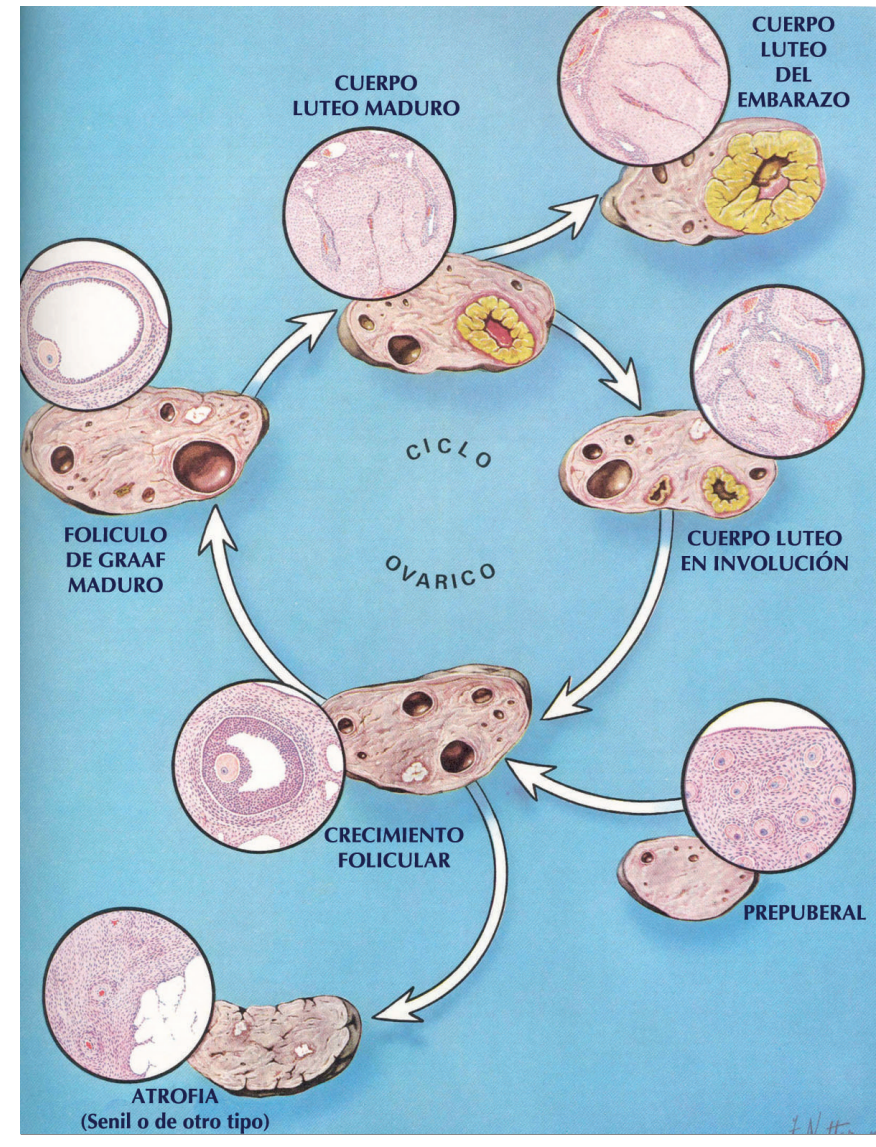

Figura 6: Fases del ciclo ovárico. Tomado de Netter (29).

convertirse en el folículo dominante. Éste agranda aún más su cavidad antral y va aproximándose a la superficie del ovario. Al final de su desarrollo alcanza un diámetro de 16 a $22 \mathrm{~mm}$ pasando a denominarse folículo maduro, terciario, de De Graaf o folículo preovulatorio.

Todo este proceso dura aproximadamente dos semanas. A partir de este momento se inician una serie de cambios que conducen a la ovulación. Se produce un despegamiento y salida del oocito de su zona de anclaje al folículo mediante la rotura folicular en su zona más prominente (estigma). Esto permite al oocito continuar su meiosis dando lugar a la formación de dos células: el oocito II y el primer corpúsculo polar.

Tras la ovulación se produce el colapso de la cavidad folicular, que se arruga y repliega y aparecen abundantes focos de hemorragia desde los vasos de la teca que dan lugar a la formación de un coágulo hemático central y a un tapón de fibrina que cierra el estroma de la ovulación. 


\section{FASE DE CUERPO LÚTEO}

Tiene una duración más constante ( $14 \pm 2$ días) y se divide en cuatro fases:

a) Proliferación: Las células de la granulosa y la teca sufren un proceso de diferenciación específica y se transforman en células luteínicas.

b) Vascularización: Se produce una gran proliferación de vasos invadiendo los espacios entre las células luteínicas.

c) Florescencia: Es la fase de máxima actividad endocrina del cuerpo lúteo, que aparece como una glándula de 17 a $20 \mathrm{~mm}$ de diámetro, de color amarillento. El culmen de su actividad se alcanza al octavo día de esta fase.

d) Regresión o involución: Si no se ha producido el embarazo, el cuerpo lúteo inicia su involución hacia el décimo día de la ovulación. Se produce una intensa infiltración por leucocitos y macrófagos, lo que implica una brusca liberación de radicales de oxígeno que, junto a otras citoquinas y factores inhibidores del crecimiento lleva a una rápida involución de la glándula. Posteriormente, todo lo que era cuerpo lúteo queda sustituido por tejido conectivo que permanece en el ovario como una cicatriz fibrosa llamada el corpus albicans.

\section{REGULACIÓN NEUROHORMONAL DEL CICLO FEMENINO (Figura 7)}

El hipotálamo es el órgano central ordenador del ritmo cíclico y cumple las funciones de "reloj biológico". Por medio de la liberación del factor liberador de gonadotropina ( $\mathrm{GnRH}$ ) provoca la liberación de gonadotropinas que actúan sobre el ovario.

Las hormonas gonadotropas son dos: la FSH u hormona estimulante de los folículos y la LH u hormona luteinizante. La FSH ejerce su acción sobre las células de la granulosa en el ovario, al mismo tiempo que estimula el crecimiento de las células germinales. La LH actúa sobre las células intersticiales y estromales promoviendo la síntesis de esteroides. Al mismo tiempo tiene una actividad fundamental en el desencadenamiento de la ovulación.

Normalmente, el primer día de hemorragia menstrual se designa como día uno del ciclo. En este momento la producción de estrógenos es baja, y la producción de FSH es máxima. Como resultado de la acción de la FSH varios folículos inician su crecimiento, aumentando los

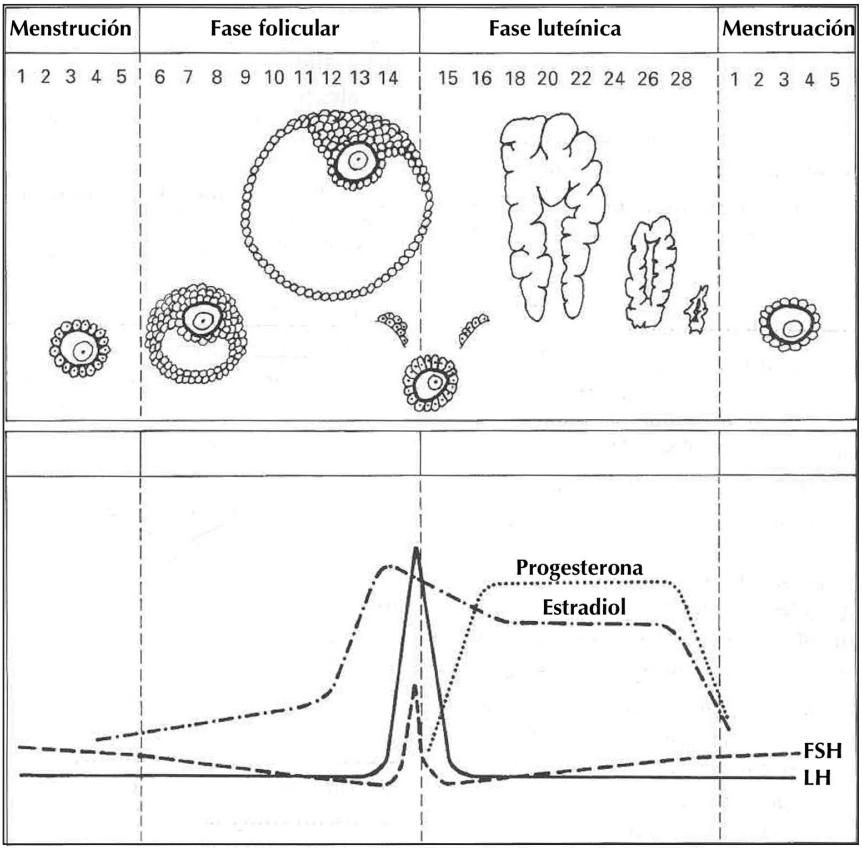

Figura 7: El ciclo ovárico en el panel superior. En el panel inferior se representan los niveles de hormonas gonadotropas y ováricas durante el ciclo. Tomada de Usandizaga (27).

niveles de estrógenos al cabo de 4 o 5 días. Durante la primera mitad del ciclo, la secreción de FSH disminuye, al tiempo que aumenta la producción de estrógenos. Esta fase estrogénica da lugar también a un aumento en la producción de LH, y, al parecer, desencadena los mecanismos endocrinos que originan la ovulación del folículo maduro en el día 14.

Después de la ovulación los niveles de estrógenos disminuyen ligeramente durante varios días. No es raro que en estos momentos aparezca una ligera hemorragia de uno o dos días de duración.

En la segunda fase del ciclo aumenta con rapidez la producción de progesterona, cuyo efecto puede detectarse fácilmente a través de los cambios que sufre el endometrio en las 48 horas siguientes a la ovulación. En este punto, la secreción de LH disminuye. Al llegar el día 20, el nivel de estrógenos es parecido al existente antes de la ovulación y la progesterona se encuentra en su fase de máxima producción. A menos que se produzca la fecundación del ovocito y que este se implante en el endometrio, se produce una disminución en la secreción de estrógenos y progesterona, lo que produce una serie de alteraciones en el endometrio, que darán lugar a su necrosis y desprendimiento (17). 


\section{REGULACIÓN NEUROHORMONAL DEL EMBARAZO}

El embarazo comienza cuando óvulo y espermatozoide se unen en la trompa. El huevo formado se traslada al útero para continuar su desarrollo hasta que el feto adquiera su capacidad para vivir en el exterior. En el caso de que se haya producido un embarazo el cuerpo lúteo no involucionará. Al decimocuarto día aumenta su tamaño y su función permanece hasta bien entrado el tercer mes de gestación. En estas circunstancias se denomina cuerpo lúteo verdadero o de embarazo (15).

El cuerpo lúteo del ovario secreta estrógenos y progesterona hasta el cuarto mes del embarazo en cantidades sólo ligeramente superiores a las que se producen después de la ovulación y en la segunda mitad del ciclo menstrual. Sin embargo, a partir del sexagésimo día de gestación, la placenta empieza a secretar estas hormonas en cantidades progresivamente elevadas, alcanzando un máximo al final del embarazo (17). Sin embargo, la placenta nunca llega a ser un órgano endocrino autónomo, ya que los esteroides producidos por ella proceden de precursores de esteroides que ingresan a través de la sangre materna o fetal.

En la biosíntesis de los estrógenos intervienen la madre, el feto y la placenta. La madre y el feto actúan como fuentes de precursores, que en la placenta son convertidos en estrógenos. Los niveles de estrógenos maternos a lo largo del embarazo alcanzarán unas concentraciones treinta veces superiores a las que se encuentran en la fase lútea.

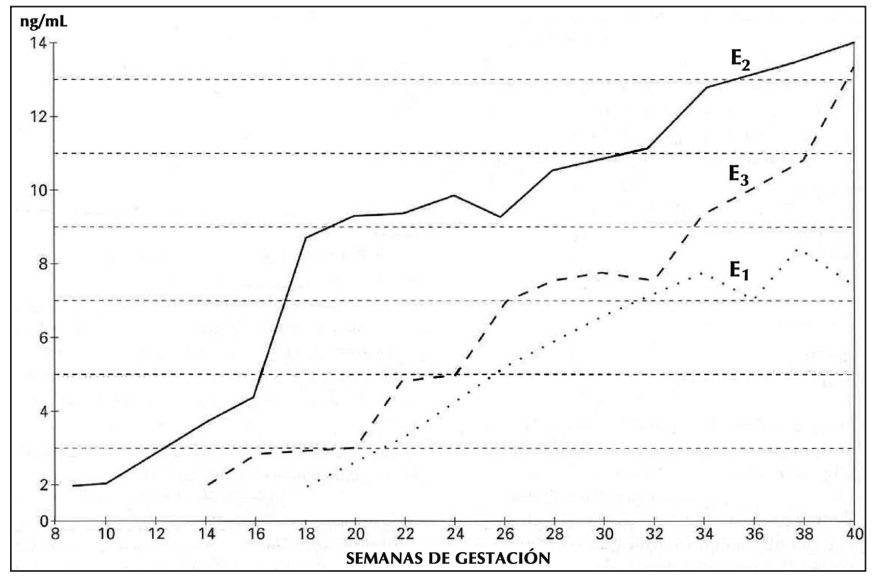

Figura 8: Variación de los niveles de estrógenos a lo largo del embarazo. E1: estrona; E2: estradiol; E3: estriol. Tomado de Usandizaga (27).
Poco después del parto la producción de estrógenos y progesterona se detiene, alcanzando valores comparables a los presentes en la mujer no embarazada (17) (Figura 8).

La progesterona se forma a través del colesterol materno. El $90 \%$ de la progesterona producida en la placenta pasa a la circulación materna y el $10 \%$ restante a la circulación fetal. Los niveles de progesterona a lo largo del embarazo aumentan progresivamente, alcanzando unas concentraciones diez veces superiores que las que se encuentran durante la fase lútea del ciclo genital. La concentración en la 27 semana de gestación es de $25 \mathrm{mg} / \mathrm{ml}$ (15) (Figura 9).

\section{PAPEL DE LAS HORMONAS SEXUALES EN LA PATOGÉNESIS DE LA GINGIVITIS GRAVÍDICA}

Aunque parece que, durante el embarazo, la susceptibilidad de los tejidos gingivales a la inflamación está relacionada con los cambios hormonales propios del mismo, el mecanismo exacto por el cual estas hormonas incrementan la inflamación gingival es desconocido. El cambio hormonal más significativo es el incremento en la producción de estrógeno y progesterona (5).

Estas hormonas ejercen su función mediante la unión a receptores intracelulares específicos que pertenecen a la superfamilia de factores de transcripción de ligandos activos que regulan el crecimiento celular, la

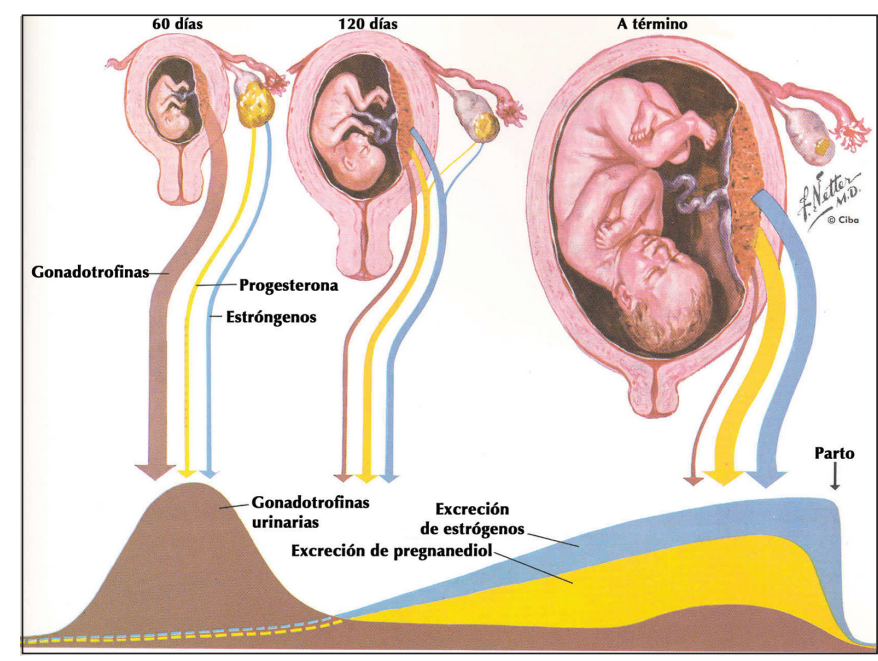

Figura 9: Variación de los niveles de estrógenos y progestágenos a lo largo del embarazo. Tomado de Netter (29). 
diferenciación y el desarrollo (18). Los estudios de ElAttar $(19,20)$ y Vittek (21) muestran que en el tejido gingival existen receptores para estrógenos y progesterona localizados en las capas basal y espinosa del epitelio gingival, en los fibroblastos del tejido conectivo y en las células endoteliales y pericitos de capilares de la lamina propia (6); Sin embargo, Parkar (18) realiza en 1996 un análisis mediante la técnica de reacción en cadena de la polimerasa (PCR) y no encuentra receptores para estrógenos y andrógenos expresados en tejidos periodontales ni gingivales, de tal modo que concluye que cualquier efecto atribuido a estas hormonas es indirecto. Esto podría explicar los datos que obtiene O'Neil (22) en su estudio en el cual no encuentra una correlación entre los niveles de hormonas circulantes y el aumento en la inflamación gingival registrado durante el embarazo. De este modo, se atribuye el efecto a los metabolitos de la progesterona y el estrógeno degradados en la encía.

En cualquier caso la respuesta de los tejidos a las hormonas sexuales femeninas va a depender del tipo de hormona circulante, de la proporción de estrógeno/progesterona así como de la concentración tisular de la hormona (5). Sus efectos a nivel del tejido gingival son variados, y tienden a ser clasificados en cuatro grandes grupos: cambios vasculares, cambios celulares, cambios microbiológicos y cambios inmunológicos $(6,8,10)$.

\section{CAMBIOS VASCULARES}

Los efectos de los esteroides a nivel vascular pueden servir para explicar la clínica de edema, eritema, sangrado e incremento de fluido crevicular gingival observada durante el embarazo. Se ha demostrado una correlación entre el aumento en el volumen del fluido crevicular gingival y unos niveles elevados de esteroides, lo que indica que las hormonas podrían alterar la permeabilidad del surco gingival (23).

Aunque a nivel general los estrógenos son las principales hormonas responsables de las alteraciones de los vasos sanguíneos en los tejidos diana de las mujeres, en el caso de la encía, parece que es la progesterona la principal responsable de los cambios vasculares. La progesterona induce la dilatación de los capilares gingivales; es responsable de la clínica de eritema y edema, del aumento del exudado capilar y la permeabilidad capilar. Todo ello debido a una acción directa sobre las células endoteliales, a variaciones en la síntesis de prostaglandinas y a la supresión de la respuesta celular asociada al embarazo $(6,8,24)$.

\section{CAMBIOS CELULARES}

Un análisis de los cambios celulares asociados refleja una disminución en la queratinización del epitelio gingival, un aumento del glucógeno epitelial, una proliferación de los fibroblastos y un bloqueo en la degradación del colágeno así como una disminución en la polimerización de la capa basal. Todo ello conduce a una disminución en la barrera epitelial y por tanto a una mayor respuesta frente a los irritantes de la placa $(6,15,24,25)$.

\section{CAMBIOS MICROBIOLÓGICOS}

Por otro lado, tanto progesterona como estrógeno aumentan el metabolismo celular de los folatos lo que contribuye a determinados cambios microbiológicos $(10,24-26)$. Se ha demostrado un aumento en la proporción de bacterias anaerobias/aerobias durante el embarazo así como un aumento en la proporción de Prevotella intermedia $(P l)$ sobre otras especies. Esta bacteria se caracteriza por requerir vitamina $\mathrm{K}$ para su crecimiento, sin embargo, es capaz de crecer en un medio suplementado con progesterona y estradiol que actúan como factores de crecimiento (26). El aumento más significativo de $P i$ se registra en el segundo trimestre del embarazo al tiempo que se observa clínicamente un aumento de la gingivitis (6). Sin embargo en un estudio desarrollado por Jonsson y cols (27) en mujeres embarazadas con enfermedad periodontal encuentran que esta patología es clínicamente semejante a la que se presenta en las mujeres no embarazadas, no evidenciando que el aumento en la concentración de hormonas en la saliva se asocie a una progresión de la enfermedad, ni a un aumento de Pi en localizaciones con periodontitis.

\section{CAMBIOS INMUNOLÓGICOS}

En cuanto a los cambios inmunológicos se ha observado una reducción en la respuesta inmune celular durante el embarazo probablemente para evitar un rechazo del cuerpo de la madre hacia el feto (28). Tanto el número como el porcentaje de linfocito T-helper (Th) disminuyen durante el embarazo y sólo se normalizan el tercer mes tras el parto. Dicho linfocitos son importantes moduladores de la respuesta inmune ya que son una fuente importante de citoquinas. Producen dos tipos funcionalmente diferentes: las citoquinas Th- 1 , encargadas de la respuesta proinflamatoria (repuesta celular), y las citoquinas Th-2 con propieda- 
des antiinflamatorias (respuesta humoral). Durante el embarazo la repuesta inmune está desviada hacia la secreción de citoquinas Th-2. Las células B y los monocitos sólo aumentan ligeramente, alcanzando el máximo en el momento de parto. Hay una disminución de la quimiotaxis de los neutrófilos y la fagocitosis $(5,6$, 29,30 ). Miyagi y cols (31) encuentran que la progesterona induce la quimiotaxis de los polimorfonucleares, mientras que el estradiol la reduce.

Se ha sugerido que la progesterona puede funcionar como un inmunosupresor en los tejidos periodontales de la mujer embarazada, evitando la aparición de una respuesta inflamatoria aguda frente al estímulo de la placa bacteriana. Esto daría lugar a la aparición de una reacción tisular crónica, con una apariencia clínica de inflamación exagerada (32).

Por ello, las reacciones inmunes locales en la encía, exacerbadas por las hormonas sexuales femeninas, pueden alterar la patogénesis de la lesión inflamatoria y con ello permitir respuestas gingivales exageradas durante el embarazo. Esta idea está soportada por el hecho de que se han identificado receptores para esteroides sexuales en componentes del sistema inmune. El-Attar $(19,20)$ descubrió que la adición de hormonas sexuales a un tejido gingival causaba un significativo incremento en la síntesis de Prostaglandina $\mathrm{E}_{2}\left(\mathrm{PG}-\mathrm{E}_{2}\right)$. Teniendo en cuenta que la PG- $\mathrm{E}_{2}$ es un potente mediador de la inflamación, este podría ser un mecanismo para explicar el papel de las hormonas sexuales en el incremento de la inflamación (33).

\section{EVIDENCIA CIENTÍIFICA}

El incremento en la severidad de las alteraciones gingivales durante el embarazo parece estar relacionado con los cambios hormonales que ocurren durante ese periodo. El mecanismo patogénico que explica este efecto aun no es conocido con exactitud, pero las observaciones realizadas por diferentes autores a lo largo de la historia apuntan hacia los cambios producidos por las hormonas sexuales femeninas a nivel vascular, celular, microbiológico e inmunológico como principales responsables del mismo $(5,6,8,24)$.

Los primeros casos de respuestas gingivales exageradas durante el embarazo fueron descritos en el siglo XIX por Eiselt en 1840 y Pinard en 1877.

Los trabajos de Löe y Silness (9) (1963) muestran que los primeros signos clínicos aparecen en el segundo mes de embarazo y continúan hasta el octavo mes, observando cierta mejoría en el último mes. Sus resultados reflejan mayor severidad de manifestaciones clínicas en el tercer y octavo mes, volviendo a la normalidad al segundo mes postparto. Encuentran que los mayores incrementos en los índices gingivales se producen en torno a los incisivos. De acuerdo con ellos, se encuentran las observaciones realizadas por RaberDurlacher (34) (1994) quien obtiene mayores valores de inflamación y sondaje en la zona anterior.

Cohen y cols $(35,36)(1969,1971)$ y Samant y cols $(37)$ (1976), encuentran por contra, que los mayores cambios en el índice gingival se producen tanto en el primero (35-37) como en el segundo trimestre del embarazo $(35,36)$, hallando mayores valores de inflamación en la mandíbula que el maxilar (35-37).

Rateitschak (38) (1967) encuentra valores de movilidad dental más pronunciados durante el embarazo que tras el parto, por lo que afirma que existe una influencia de las hormonas sexuales femeninas en la membrana periodontal. Sin embargo, Hugoson (23) (1970) y Lundgren y Lindhe (39) (1971), demuestran que el agravamiento de la gingivitis durante el embarazo no supone daños permanentes en las porciones más profundas del periodonto, por lo que sugieren que el incremento en la movilidad dental observado por Rateitschak no se debe a una pérdida de soporte óseo sino a cambios cualitativos en el ligamento periodontal.

Uno de los primeros estudios que sugiere una correlación entre el nivel de hormonas y la severidad de la gingivitis es el de Ziskin y col (40) (1933). Desde entonces múltiples estudios han comprobado esta evidencia y han establecido una etiología multifactorial en relación con el sistema endocrino para poder explicar estos cambios.

Ziskin y col. (1933) (40), Nutlay y col. (41) (1954) y Shkalr y col. (42) (1956) afirman que existen múltiples efectos histológicos visibles en el epitelio y conectivo gingival tras administrar hormonas sexuales en animales ovariectomizados. Por el contrario, Litwack y col. (43) (1970), Rubright y col. (44) (1971) y Hugoson y Lindhe $(45,46)(1971)$ no observan dichos efectos.

Turesky y col. (47) (1958) postulan que las hormonas sexuales actúan directamente sobre la encía, mientras que Löe y Silness (9) (1963) sugieren que la función de las hormonas sexuales es modificar la respuesta inflamatoria de la encía a los irritantes locales. 
Lindhe y col (48) (1967) en un estudio experimental en hámster analizan los efectos de las hormonas sobre el sistema vascular comprobando que estas inducen un impedimento vascular, por lo que concluyen que la gingivitis del embarazo puede ser considerada como una reacción tisular en la que las hormonas sexuales actúan sobre el tejido y el sistema vascular disminuyendo el nivel umbral a partir del cual distintos agentes son capaces de inducir daño tisular gingival. Este mismo autor $(49,50)$, en 1968, estudia la influencia de las hormonas sexuales en la exudación gingival en perros hembra con y sin gingivitis. En ambos casos se produce un incremento del volumen de fluido crevicular gingival que se recupera una vez que cesa la administración de hormonas, lo que explican por un aumento de la permeabilidad de los vasos dentogingivales.

El-Attar y cols $(19,20)(1973,1974)$ en un estudio de experimentación animal proponen que la hiperactividad de los sistemas enzimáticos esteroideos específicos y la acumulación de sus metabolitos en la encía puede ser un factor influyente en la etiología de la gingivitis gravídica.

Recientemente se ha aceptado una nueva clasificación de las enfermedades periodontales dentro de las que se incluyen las enfermedades gingivales asociadas al embarazo (gingivitis gravídica y granuloma piogénico) como una patología inducida por placa y modificada por factores sistémicos (International Workshop Classification for Periodontal Diseases, 1999) (51). Se establece que las hormonas sexuales no son necesarias ni suficientes para producir cambios gingivales por si mismas, sin embargo son capaces de alterar la respuesta de los tejidos gingivales a la placa dental y de esta forma contribuir indirectamente al desarrollo de la enfermedad periodontal.

\section{APLICACIÓN PRÁCTICA: UTILIDAD DE LA SALIVA COMO MEDIO DIAGNÓSTICO}

La saliva constituye una muestra biológica de fácil obtención (no invasiva), de bajo coste e indolora, cuya composición puede reflejar en gran medida ciertos acontecimientos de manifestación sistémica. La Academia de Ciencias de Nueva York, el Instituto Nacional de Investigación Dental de Estados Unidos y numerosos grupos de investigación sobre saliva han apoyado y recomiendan maximizar el potencial de este fluido para su uso en investigación, con el fin de facilitar el diagnóstico y monitorizar el estado de salud general y bucodental de la población general $(52,53)$.

En los años ochenta se demostró que la saliva contenía hormonas (54-56). Los estudios han permitido establecer que el nivel de una hormona en saliva se correlaciona con el nivel libre de esa hormona en sangre (57). Las hormonas esteroides en saliva son excepcionalmente estables y pueden ser medidas exactamente. En muchos estudios las concentraciones de estradiol y progesterona fueron medidas mediante radioinmunoanálisis en saliva, utilizando estos datos como indicador importante de la fecundidad.

La determinación de las concentraciones salivales de progesterona se ha utilizado extensamente puesto que proporciona un medio de determinación de la función ovárica durante el ciclo y el embarazo temprano. Los estudios encuentran correlaciones significativamente altas entre la progesterona total y libre en plasma y la progesterona salival $(54,58,59)$.

Los niveles de estradiol en saliva se pueden utilizar para determinar la dinámica folicular, sin embargo existen limitaciones metodológicas a la hora de analizarlos, lo que hace que su uso esté menos extendido $(55,60)$.

El papel de las hormonas en el desarrollo de alteraciones gingivales durante el embarazo ha sido estudiado por diferentes autores, encontrando resultados contradictorios entre algunos de ellos. O'Neil (22) realiza un estudio con 26 mujeres embarazadas (semana 14-30 de gestación) y no encuentra una relación directa entre las concentraciones de progesterona y estradiol medidas en plasma y los valores de índice gingival, por lo que propone un efecto indirecto de los metabolitos sobre el tejido gingival.

Estos resultados están de acuerdo con los presentados por Muramatsu y cols (61), que, estudian a un grupo de 19 mujeres embarazadas, 12 mujeres no embarazadas y 8 mujeres en el quinto mes tras el parto. Analizan $1 \mathrm{ml}$ de saliva de parótida estimulada y analizan las concentraciones de estradiol y progesterona mediante radioinmunoanálisis. Obtienen unos valores de progesterona de $0,13 \mathrm{ng} / \mathrm{ml}$ en el primer trimestre y $0,24 \mathrm{ng} / \mathrm{ml}$ en el segundo. Sin embargo no consiguen establecer una correlación entre ellas y el grado de inflamación gingival.

Jonsson y cols (27) (1988) en un estudio con embarazadas con enfermedad periodontal previa no encuen- 
tran correlación entre los parámetros clínicos y los niveles de esteroides en saliva.

Por otro lado Vittek y cols (21) sí que encuentran una relación entre la concentración de esteroides en saliva y un incremento en la incidencia de enfermedad periodontal. Sin embargo los sujetos de su estudio poseen periodontitis avanzada. Zaki y cols (62) realizan un estudio con 30 embarazadas (10 en cada trimestre del embarazo) y 10 mujeres no embarazadas en la fase lútea (17-2l días del ciclo) en el que valoran las concentraciones de progesterona y $17 \mathrm{~b}$-estradiol en muestras de saliva no estimulada mediante radioinmunoanálisis. Obtienen valores de progesterona de 220$280 \mathrm{pg} / \mathrm{ml}$ para el primer trimestre y $460-1590 \mathrm{pg} / \mathrm{ml}$ para el segundo trimestre. Al mismo tiempo encuentran un incremento en la severidad de la inflamación gingival según avanza el embarazo, por lo que concluyen que el aumento de la concentración de hormonas en saliva es un factor etiológico importante de la alteración gingival asociada al embarazo.

\section{CONCLUSIONES}

1. Durante el embarazo se produce una alteración en los niveles de hormonas sexuales femeninas que conlleva la aparición de manifestaciones sistémicas propias del mismo.

2. Los cambios hormonales asociados al embarazo parecen ser los responsables del desarrollo de la gingivitis gravídica, aunque el mecanismo patogénico responsable aun es desconocido.

3. A lo largo de la historia diferentes autores han tratado de encontrar una correlación entre los cambios en los niveles de hormonas sexuales femeninas y el desarrollo de alteraciones gíngivo-periodontales; sin embargo los resultados obtenidos son contradictorios.

4. El empleo de la saliva como medio diagnóstico ha facilitado el estudio de los niveles hormonales de las pacientes embarazadas.

5. Son necesarios más estudios que determinen el papel exacto de las hormonas sexuales femeninas en los tejidos gíngivo-periodontales.

\section{ABSTRACT}

Pregnancy gingivitis is a proliferative, vascular and non-specific inflammation accompanied by a wide cellular inflammatory infiltrate. Clinically it is characterized by an increased gingival redness and bleeding on probing index, gingival margin augmentation and hyperplasia of the interdental papillae. It is extremely common and affects $30-100 \%$ of all pregnant women.

Even though it seems that during pregnancy susceptibility of gingival tissues to inflammation is related to altered hormonal levels, the exat mechanism by which they exert their effects remain unknown. The most significant hormonal change is the increase in the production of estrogens and progesterone.

Tissue's response to female sex hormones depends on the circulating hormone type, the estrogens/progesterone ratio and on the tissular concentration of the hormone. Their effects on the gingival tissues are diverse and tend to be classified into four main groups: vascular, cellular, microbiological and immunological changes.

\section{KEY WORDS}

Gingivitis. Pregnancy. Pyogenic granuloma. Estrogens. Progesterone. Pathogenesis. Diagnosis. Pegnancy gingivitis.

\section{BIBLIOGRAFÍA}

1. Grau DM, Silvestre FJ, Miralles L, Roig JM. La secreción salival durante el embarazo. Rev Eur Odontoestomatol 2002;XIV(2):93-8.

2. Arafat AH. Periodontal status during pregnancy. J Periodontol 1974;45(8):641-3.

3. Chaikin BS. Incidence of gingivitis in pregnancy. Quintessence International 1977;8(10):81-9.

4. Conde Vidal J, Inglés Castello M, Purificación Roldán G. Enfermedad parodontal en el embarazo. Revista Española de Estomatología 1981;29(3):179-90.

5. Laine MA. Effect of pregnancy on periodontal and dental health. Acta Odontol Scand 2002; 60:257-64.

6. Garrido de Cab N, Blanco Carrión J, Ramos Barbosa I. Enfermedad periodontal y embarazo. Periodoncia 1999;9(1):31-40.

7. Gaffield ML, Colley Gilbert BJ, Malvitz DM, Romaguerra R. Oral health during pregnancy. An analysis of 
information collected by the Pregnancy Risk Assessment Monitoring System. JADA 2001;132:100916.

8. Mealy BL, Moritz, AL. Hormonal influences: effects of diabetes mellitus and endogenous female sex steroid hormones on the periodontium. Periodontology 2000 2003;32:59-81.

9. Löe H \& Silness J. Periodontal disease in pregnancy: prevalence and severity. Acta Odontologica Scandinavia 1963;21:533-51.

10. Zachariasen RD. The effect of elevated ovarian hormones on periodontal health: oral contraceptives and pregnancy. Women \& Health 1993;20(2):21-30.

11. Bascones A, Llanes F. Medicina Bucal. Tomo I. $2^{\mathrm{a}}$ Edición. Avances ed. Madrid, 1991: 274.

12. Ojanotko-Harri AO, Harri M-P, Hurttia HM, Sewon LA. Altered tissue metabolism of progesterone in pregnancy gingivits and granuloma. J Clin Periodontol 1991;18:262-6.

13. Yuan $\mathrm{K}$, Wing LC, Lin M. Pathogenetic roles of angiogenic factors in pyogenic granulomas in pregnancy are modulated by female sex hormones. J Periodontol 2002;73:701-8.

14. Silverstein LH, Burton C, Garnick J, Singh B. The late development of oral pyogenic granuloma as a complication of pregnancy: a case report. Compendium 1996;17(2):192-8.

15. Usandizaga JA, De la Fuente P. Tratado de obstetricia y Gnecología. Volumen I. Ed McGraw-Hill. Interamericana. Madrid, 1997.

16. González- Merlo J, González Bosquet J, González Bosquet E. Ginecología. Ed Masson. $8^{a}$ edición. Barcelona, 2003.

17. Netter FH. Sistema reproductor. Tomo 2. Ed Salvat. Barcelona, 1990.

18. Parkar MH, Newman HN, Olsen I. Polymerase chain reaction analysis of oestrogen and androgen receptor expression in human gingival and periodontal tissue. Archs oral Biol 1996;41(10):979-83.

19. El Attar TMA, Hugoson A. Comparative metabolism of female sex steroids in normal and chronically inflamed gingiva of the dog. J Periodontal Res 1973;9(5):284-9.
20. El Attar TMA, Roth GD, Hugoson A. Comparative metabolism of $4-{ }^{14} \mathrm{C}$-progesterone in normal and chronically inflamed human gingival tissue.J Periodont Res 1973;8(2):79-85

21. Vittek J, Rappaport SC, Gordon GG, Munnangi PR, Southren AL. Concentration hormones and metabolism of androgens by human gingiva. J Periodontol 1979;50(5):254-64.

22. O'Neil TCA. Plasma female sex-hormone levels and gingivitis in pregnancy. J Periodontol 1979;50(6):279-82.

23. Hugoson A. Gingival inflammation and female sex hormones. J Periodont Res 1970;5(suppl):1-18.

24. Sooriyamoorthy M, Gower DB. Hormonal influences on gingival tissue: relationship to periodontal disease. J Clin Periodontol 1989;16:201-8.

25. Deasy MJ, Grota LJ, Kennedy JE. The effect of estrogen, progesterone and cortisol on gingival inflammation. J Periodont Res 1972;7:111-24

26. Kornman $\mathrm{K}$, Loesche $\mathrm{W}$. The subgingival microbial flora during pregnancy. J Period Res 1980;15:111-22.

27. Jonsson R, Howland BE, Bowden GHW. Relationship between periodontal health, salivary steroids and Bacteroides intermedius in males, pregnant and nonpregnant women. J Dent Res 1988;67(8):1062-9.

28. O'Neil TCA. Maternal T-Lymphocyte response and gingivitis in pregnancy. J Periodontol 1979;50(4):178-84.

29. Raber-Durlacher JE, Zeijlemaker WP, Meinesz AAP, Abraham-Inpijn $\mathrm{L}$. CD4 to CD8 ratio and in vitro lymphoproliferation responses during experimental gingivitis in pregnancy and post-partum. J Periodontol 1991;62:663-7.

30. Heasman PA, Collins JG, Offenbacher S. Changes in crevicular fluid levels of interleukin-lb, leukotriene $B_{4}$, prostaglandin $\mathrm{E}_{2}$, thromboxane $\mathrm{B}_{2}$ and tumor necrosis factor a in experimental gingivitis in humans. J Periodont Res 1993;28:241-7.

31. Miyagi M, Morishita M, Iwamoto Y. Effects of sex hormones on production of prostaglandin $E_{2}$ by human peripheral monocytes. J Periodontol 1993;64:1075-8.

32. Tilakaratne A, Soory M. Effects of the anti-androgen finasteride on 5a-reduction of androgens in the presence of progesterone in human gingival fibroblasts: 
modulatory actions of the alkaline phosphatase inhibitor levamisole. J Periodont Res 2000; 35:179-85.

33. Yaldin F, Basegmez C, Gulden I, Berber L, Eskinazi E, Soydine M. et al. The effects of periodontal therapy on intracrevicular prostaglandin $\mathrm{E}_{2}$ concentrations and clinical parameters in pregnancy.J Periodontol 2002;73: 173-7.

34. Raber-Durlacher JE, Van Steenbergen TJM, Van der Velden U, de Graaff J, Abraham-Inpijn L. Experimental gingivitis during pregnancy and post-partum: clinical, endocrinological, and microbiological aspects. J Clin Periodontol 1994;21:549-58.

35. Cohen W, Friedman L, Shapiro J, Kyle GC. A longitudinal investigation of the periodontal changes during pregnancy. J Periodontol 1969;40:563-70.

36. Cohen W, Friedman L, Shapiro J, Kyle GC, Franklin S. A longitudinal investigation of the periodontal changes during pregnancy and fifteen months post-partum. Part II. J Periodontol 1971;42(10):653-7.

37. Samant A, Malik CP, Chabra SK, Devi PK. Gingivitis and periodontal disease in pregnancy. J Periodontol 1976;47(7):415-8.

38. Rateitschak $\mathrm{KH}$. Tooth mobility changes in pregnancy. J Periodontal Res 1967;2(3):199-206.

39. Lundgren $D$, Lindhe J. Lack of influence of female sex hormones on alveolar bone loss in hamsters. Scand J Dent Res 1971;79:113-8.

40. Ziskin DE, Blackberg SN, Stout AP. The gingiva during pregnancy. AN experimental study and a histopathological interpretation. Surg Gynecol Obstet 1933;57: 119.

41. Nutlay AG, Bhaskar SN, Weinmann JP, Budy AM. The effect of estrogen on the gingival and alveolar bone of molars in rats and mice. J Dent Res 1954;33(1):115-27.

42. Shkalr G, Glickman I. The effect of estrogenic hormone on the periodontium of white mice. J Periodont 1956; 27:16-23.

43. Litwack D, Kennedy JE, Zander HA. Response of oral epithelia to ovariectomy and estrogen replacement. J Periodontal Res 1970;5(4):263-8.

44. Rubright WC, Higa LH, Yannone ME. Histological quantification of the biological effects of estradiol benzoate on the gingival and genital mucosa of castrated rabbits. J Periodontal Res 1971;6(1):55-64.

45. Hugoson $A$ \& Lindhe J. Gingival tissue regeneration in female dogs treated with sex hormones. Histological observations. Odontol Revy $1971 ; 22(4)$ :425-39.

46. Hugoson $A$ \& Lindhe J. Gingival tissue regeneration in female dogs treated with sex hormones. Clinical observations. Odontol Revy 1971;22(3):237-49.

47. Turesky S, Fisher B, Glickman I. A histochemical study of the attached gingival in pregnancy. J Dent Res 1958;37(6):1115-22.

48. Lindhe J, Branemark PI. Changes in microcirculation after local application of sex hormones. J Periodont Res 1967;2:185-93.

49. Lindhe J, Attstrom R, Björn A. Influence of sex hormones on gingival exudation in gingivitis-free female dogs. J Periodont Res 1968;3:273-8.

50. Lindhe J, Attstrom R, Björn A. Influence of sex hormones on gingival exudation in dogs with chronic gingivitis. J Periodont Res 1968;3:279-83.

51. Armitage GC. Development of a classification system for periodontal diseases and conditions. Ann Periodontol 1999;4:1-6.

52. Medina M, Merino LA, Gorodner JO. Utilidad de la saliva como fluido diagnóstico en http://www. webodontologica.com/odon_arti_uti_saliv.asp.

53. Riad-Fahmy D, Read GF, Walker RF, Griffiths K. Steroids in saliva for assessing endocrine function. Endocrine Rev 1982;3:367-95.

54. Sufi SB, Donaldson A, Gandy SC, Jeffcoat SL, Chearskul $\mathrm{S}$, Gon $\mathrm{H}$ et al. Multicenter evaluation of assays for estradiol and progesterone in saliva. Clin Chem 1985;31 (1):101-3.

55. Belkien LD, Bordt J, Moller P, Hano R, Nieschlag E. Estradiol in saliva for monitoring follicular stimulation in an in vitro fertilization program. Fertil Steril 1985;44(3):322-7.

56. Berthonneau J, Begon F, Bounaud JY, Chansigaud JP, Cedard L. Evolution of salivary estradiol levels during the spontaneous menstrual cycle. Correlation between saliva and plasma.J Gynecol Obstet Biol Reprod (Paris) 1989;18(1);47-52. 
57. Marek B, Kot T, Bunter B. Usefulness of measuring the levels of estrogens and progesterone in the saliva during pregnancy. Ginekol Pol 1989; 60(5):291-4.

58. Wong YF, Mao K, Panesar NS, Loong EP, Chang AM, Mi ZJ. Salivary estradiol and progesterone during the normal ovulatory menstrual cycle in Chinese women. Eur J Obstet Gynecol Reprod Biol 1990;34(1-2):129-35.

59. Meulenberg EP, Hofman JA. The effect of pretreatment of saliva on steroid hormone concentrations.J Clin Chem Clin Biochem 1990;28(12):923-8.
60. Worthman CM, Stallings JF, Hofman LF. Sensitive salivary estradiol assay for monitoring ovarian function. Clin Chem 1990;36(10):1769-73.

61. Muramatsu Y, Takaesu Y. Oral health status related to subgingival bacterial flora and sex hormones in saliva during pregnancy. Bull Tokyo dent Coll 1994;35(3):13951.

62. Zaki H, Rl Hak R, Amer W, Saleh F, El Faras A, Ragab L et al. Salivary female sex hormone levels and gingivitis in pregnancy. Biomed Biochim Acta 1984;43(6):749-54. 